\title{
Robust MIMO Radar Waveform Design with Imperfect Prior Knowledge of Targets
}

\author{
Yuchun Liu ${ }^{1,2}$, Hai Zhu ${ }^{3}$ and Hongyan Wang ${ }^{4}$ \\ ${ }^{1}$ School of Physics and Electrical Engineering, Zhoukou Normal University, \\ Zhoukou, 466001, China \\ ${ }^{2}$ National Key Laboratory of Radar Signal Processing, Xidian University, Xi'an, \\ 710071, China \\ ${ }^{3}$ School of Computer Science and Technology, Zhoukou Normal University, \\ Zhoukou, 466001, China, \\ ${ }^{4}$ College of Information Engineering, Dalian University, Dalian, 116622, China, \\ E-mail:lycdgp@163.com,Email:Zhu_sea@163.com,E-mail:gglongs@163.com
}

\begin{abstract}
Waveform design for multi-input multi-output (MIMO) radar, which usually relies on the initial parameter estimates (i.e., some prior information on the target of interest and scenario), is often sensitive to estimation errors in these parameters. To systematically alleviate this sensitivity, robust waveform design has been studied in recent years by explicitly incorporating a parameter uncertainty model into the optimization problem. In this paper, the robust MIMO radar waveform optimization is investigated to improve the worst-case performance of parameter estimation over a convex uncertainty model, which is based on the Cramer-Rao bound (CRB). To solve the resultant complicated nonlinear optimization problem, an iterative algorithm is proposed to optimize the waveform covariance matrix (WCM) such that the worst-case performance can be improved. Each iteration step in the proposed algorithm can be relaxed as a semidefinite programming (SDP) problem by using the associated matrix inequality. Numerical results show that the worst-case parameter estimation performance can be improved considerably by the proposed method compared to uncorrelated waveforms.
\end{abstract}

Keywords: Multi-input multi-output (MIMO) radar, robust waveform design, convex relaxation, parameter estimation, semidefinite programming (SDP)

\section{Introduction}

Radar system employing multiple antennas on both transmitter and receiver has been intensively researched in the last decade, which is the so-called multi-input multi-output (MIMO) radar [1]-[13]. MIMO radar has the flexibility to transmit diverse waveforms from its different transmit antenna elements, which is the so-called waveform diversity [1]. In terms of the spacing between its antennas, MIMO radar can be classified into two categories shown as: (1) MIMO radar with widely separated antennas (e.g. [2]), and (2) MIMO radar with colocated antennas (e.g. [1]). The former employs widely-spaced the transmitting and receiving elements along with diverse transmitted waveforms to view the different aspects of the target thereby improving the target detection performance. In contrast, the latter employs close-spaced elements in transmitting and receiving arrays to obtain the identical target radar cross sections (RCSs) observed from all transmit/receive paths, which can utilize the waveform diversity to increase the virtual aperture of the receiving array [1]. Accordingly, it has several advantages including improved parameter identifiability [3], and more flexibility for transmit beampattern design [4]-[12]. In this paper, we consider MIMO radar with collocated antennas. 
A particularly critical issue for both types of MIMO radar is the waveform optimization [4]-[12], which has received considerable attention recently. According to the objects needed to be optimized, the current design methods can be divided into two categories: (1) only the transmitter to be designed [4]-[6], and (2) the transmitter and receiver to be designed jointly [7]-[12]. In [4], the waveform covariance matrix (WCM) was designed to attain a desired beam pattern, while the constant modulus signal design is considered in [5]. Meanwhile, the spatial-range-Dopper domain characteristics of the transmitted waveforms were considered jointly in [6]. In [7], the transmit waveforms were optimized for multiple point targets based on several design criteria, for example, minimizing the trace of the Cramér-Rao Bound (CRB) matrix. The output signal-tointerference-plus-noise ratio (SINR) was maximized in [8] to improve the detection performance for MIMO radar. Unfortunately, the method proposed in [8] cannot guarantee nondecreasing SINR in each iteration step. In order to guarantee convergence, a new iterative algorithm was derived in [9].The mutual information between the received waveforms and the target radar signatures was employed to design the transmit waveform for extended target [10]. In [11], MIMO waveform was devised by minimizing the estimation error of the minimum mean squared error (MMSE). The joint optimization of the WCM and receiver is investigated to improve the parameter estimation performance in [12].

In [7], the problem of MIMO radar waveform design for parameter estimation of point targets has been investigated, which is based on the Cramer-Rao bound (CRB). It is obvious that the optimization problem to be solved in this document requires the specification of parameters, e.g., the target location, reflection coefficients, etc.. However, these parameters cannot be obtained directly and must be estimated in practice, and hence they are uncertain. As illustrated by numerical examples in [7], the resultant accuracy of parameter estimation is sensitive to these estimation errors and uncertainty in parameters. It means that the optimized waveforms based on a certain parameter estimate can give a very low performance of parameter estimation for another reasonable estimate.

In this paper, the problem of robust waveform design to improve the worst-case estimation performance of MIMO radar is investigated based on CRB, which attempts to systematically alleviate the sensitivity by explicitly incorporating a convex parameter uncertainty model in the optimization issue. To solve the resultant complicated nonlinear optimization problem, an iterative algorithm is proposed to design the WCM such that the worst-case performance can be improved. By using some matrix inequalities, each step in the proposed algorithm can be relaxed as a semidefinite programming (SDP) problem [13], and hence it can be solved efficiently.

The rest of this paper is organized as follows. Section 2 introduces the MIMO radar model, gives the derivation of the CRB, and formulates the robust waveform optimization problem. Section 3 proposes an iteration algorithm and recasts each iteration step as the convex relaxation formulation. Section 4 shows the effectiveness of the proposed method via numerical examples. Finally, Section 5 concludes this paper.

Throughout the paper, matrices and vectors are denoted by boldface uppercase and lowercase letters, respectively. We use $\{\cdot\}^{T},\{\cdot\}^{*}$, and $\{\cdot\}^{H}$ to represent the transpose, conjugate, and conjugate transpose, respectively. The symbol $\otimes$ indicates the Kronecker product, I denotes the identity matrix, and $\operatorname{vec}\{\cdot\}$ is the vectorization operator stacking the columns of a matrix on top of each other. The notation $\|\mathbf{A}\|_{F}$ stands for the Frobenius norm of the matrix $\mathbf{A}$, and $\operatorname{diag}\{\mathbf{a}\}$ for a diagonal matrix with its diagonal given by the vector $\mathbf{a}$. Denote by $\operatorname{tr}\{\cdot\}, \operatorname{Re}\{\cdot\}$ and $\operatorname{Im}\{\cdot\}$ the trace, the real and imaginary part of a matrix, respectively. Given a vector function $\mathbf{f}: \square^{n} \rightarrow \square^{k}$, we denote by $\frac{\partial \mathbf{f}}{\partial \boldsymbol{\theta}}$ the $k \times n$ 
matrix whose ijth element is $\frac{\partial \mathbf{f}_{i}}{\partial \boldsymbol{\theta}_{j}}$. Finally, the notation $\mathbf{A}^{\circ} \mathbf{B}$ means that $\mathbf{B}-\mathbf{A}$ is positive semidefinite.

\section{Problem Formulation}

The signal model adopted in this paper is the same as that illustrated in [7], and the signals received by MIMO radar can be expressed as:

$$
\mathbf{Y}=\sum_{k=1}^{K} \beta_{k} \mathbf{H}_{k} \mathbf{S}+\mathbf{W}
$$

where $\mathbf{S}=\left[\mathbf{s}_{1}, \mathbf{s}_{2}, \cdots, \mathbf{s}_{M_{t}}\right]^{T} \in \square^{M_{t} \times L}$ be the transmitted waveform matrix with $\mathbf{s}_{i} \in \square^{L \times 1}$ denoting the discrete-time baseband signal of the ith transmitting element along with $L$ being the number of snapshots. $\left\{\beta_{k}\right\}_{k=1}^{K}$ are the complex amplitudes proportional to the RCSs of these targets, and $\left\{\theta_{k}\right\}_{k=1}^{K}$ denote those location parameters with $K$ being the number of targets of interest, both of which need to be estimated from the received signal $\mathbf{Y} \in \square^{M_{r} \times L}$. The term $\mathbf{W}$ is the noise plus interference, similar to that illustrated in [7], whose columns can be assumed to be independent and identically distributed circularly symmetric complex Gaussian random vectors with mean zero and an unknown covariance denoting by $\mathbf{Q}$. Also, $\mathbf{H}_{k}=\mathbf{a}\left(\theta_{k}\right) \mathbf{v}^{T}\left(\theta_{k}\right)$ represents the kth target channel matrix. Besides, $\mathbf{a}\left(\theta_{k}\right)$ and $\mathbf{v}\left(\theta_{k}\right)$ denote, respectively, the receiving and transmitting steering vectors for the target located at $\theta_{k}$, which can be described as

$$
\begin{aligned}
& \mathbf{a}\left(\theta_{k}\right)=\left[e^{j 2 \pi f_{0} \tau_{1}\left(\theta_{k}\right)}, e^{j 2 \pi f_{0} \tau_{2}\left(\theta_{k}\right)}, \cdots, e^{j 2 \pi f_{0} \tau_{M_{r}}\left(\theta_{k}\right)}\right]^{T} \\
& \mathbf{v}\left(\theta_{k}\right)=\left[e^{j 2 \pi f_{0} \bar{\tau}_{1}\left(\theta_{k}\right)}, e^{j 2 \pi f_{0} \bar{\tau}_{2}\left(\theta_{k}\right)}, \cdots, e^{j 2 \pi f_{0} \bar{\tau}_{M_{t}}\left(\theta_{k}\right)}\right]^{T}
\end{aligned}
$$

where $f_{0}$ represents the carrier frequency. $\tau_{m}\left(\theta_{k}\right), m=1,2, \cdots M_{r}$ is the propagation time from the target located at $\theta_{k}$ to the mth receiving element, and $\tilde{\tau}_{n}\left(\theta_{k}\right), n=1,2, \cdots M_{t}$ is the propagation time from the nth transmitting element to the target.

We now consider the CRB of the unknown parameters $\boldsymbol{\theta}=\left[\theta_{1}, \theta_{2}, \cdots, \theta_{K}\right]^{T}$ for the case of known $\left\{\beta_{k}\right\}_{k=1}^{K}$, which is the so-called constrained CRB [14]. The constrained CRB is derived in Appendix A, which can be expressed as

$$
\mathbf{C}_{C C R B}=\left[\begin{array}{cc}
\mathbf{C}_{11} & \mathbf{0}_{K \times 2 K} \\
\mathbf{0}_{2 K \times K} & \mathbf{0}_{2 K \times 2 K}
\end{array}\right]
$$

where

$$
\mathbf{C}_{11}=\left(2 \operatorname{Re}\left(\mathbf{F}_{11}\right)\right)^{-1}
$$

and

$$
\left[\mathbf{F}_{11}\right]_{i j}=\operatorname{tr}\left[\beta_{i}^{*} \beta_{j} \dot{\mathbf{H}}_{i}^{H} \mathbf{Q}^{-1} \dot{\mathbf{H}}_{j} \mathbf{R}_{\mathbf{S}}\right]
$$

in which $\mathbf{R}_{\mathbf{S}}=\mathbf{S S}^{H}$, and $\dot{\mathbf{H}}_{k}=\frac{\partial \mathbf{H}_{k}}{\partial \theta_{k}}, k=1,2, \cdots, K$.

It can be seen from (5) that the constrained CRB is a function with respect to the location $\theta$ as well as the noise plus interference. In practice, these parameters are estimated with errors and so they are uncertain. Hence, the optimized waveforms based on the CRB employing a parameter estimate can give a very low accuracy for another reasonable estimate, which has been illustrated via numerical examples in [7]. 
Here, we assume that the derivation of the kth target channel matrix is uncertain, but known to belong to a convex compact set, which can be modeled as:

$$
\dot{\tilde{\mathbf{H}}}_{k}=\dot{\mathbf{H}}_{k}+\dot{\boldsymbol{\delta}}_{k}, k=1,2, \cdots, K
$$

where $\dot{\tilde{\mathbf{H}}}_{k}$ and $\dot{\mathbf{H}}_{k}$ are, respectively, the derivation of the kth actual target channel matrix and the corresponding presumed target channel matrix, and $\dot{\boldsymbol{\delta}}_{k}$ denotes the error of $\dot{\tilde{\mathbf{H}}}_{k}$, which belongs to the set

$$
\mathrm{U}=\left\{\dot{\boldsymbol{\delta}}_{k} \mid\left\|\dot{\boldsymbol{\delta}}_{k}\right\|_{F} \leq \sigma_{k}, k=1,2, \cdots, K\right\}
$$

We assume that $\sigma_{k}<\left\|\dot{\mathbf{H}}_{k}\right\|_{F}$ in this paper.

The robust waveform optimization for improving the performance of parameter estimation can now be briefly stated as follows: Optimize the WCM to minimize the worst-case CRB over the convex set U under the constraints about the WCM. Under the Trace-opt criterion [7], this optimization problem can be illustrated as

$$
\begin{aligned}
\min _{\mathbf{R}_{\mathbf{S}}} & \max _{\left\{\delta_{k}\right\}_{k=1}^{K}} \\
\text { s.t. } & \operatorname{tr}\left(\mathbf{C}_{C C R B}\right) \\
& \dot{\boldsymbol{\delta}}_{k} \in \mathrm{U} \\
& \operatorname{tr}\left(\mathbf{R}_{\mathbf{S}}\right)=L P \\
& \mathbf{R}_{\mathbf{S}} \pm \mathbf{0}
\end{aligned}
$$

where $P$ denotes the total transmitted power. The third constraint holds due to the power transmitted by any transmitting element is more than or equal to zero in practice.

It is obvious that the trace of the objective in (8), is a rather complicated nonlinear function of $\mathbf{R}_{\mathrm{s}}$ and $\dot{\boldsymbol{\delta}}_{k}, k=1,2, \cdots, K$. Consequently, the problem is difficult to be solved by using the tradition method, for example, the convex optimization method [13].

\section{Proposed Iterative Method}

In this section, we demonstrate how to obtain an optimal solution of the nonlinear optimization problem in (8). For this purpose, we first consider the inner optimization problem, i.e., solving $\dot{\boldsymbol{\delta}}_{k}$ in terms of $\mathbf{R}_{\mathbf{s}}$. In order to solve it, we can rely on the following lemma [16]:

Lemma 1: Let A be a $M \times M$ positive semidefinite Hermitian matrix, then the following inequalities

$$
\operatorname{tr}\left(\mathbf{A}^{-1}\right) \geq \sum_{i=1}^{M} \frac{1}{a_{i i}}
$$

holds, where the equality is achieved if and only if $\mathbf{A}$ is diagonal.

According to Lemma 1, the maximization in (8) can be relaxed to

$$
\begin{aligned}
\max _{\left\{\dot{\delta}_{k}\right\}_{k=1}^{K}} & \sum_{k=1}^{K}\left(2\left|\beta_{k}\right|^{2} \operatorname{tr}\left[\dot{\tilde{\mathbf{H}}}_{k}^{H} \mathbf{Q}^{-1} \dot{\tilde{\mathbf{H}}}_{k} \mathbf{R}_{\mathbf{S}}\right]\right)^{-1} \\
\text { s.t. } & \dot{\boldsymbol{\delta}}_{k} \in \mathrm{U}
\end{aligned}
$$

omitting the operator $\operatorname{Re}\{\cdot\}$ due to every term in the summation is a real number. It can be seen from (10) that the $k$ th term in the summation only depends on $\dot{\boldsymbol{\delta}}_{k}$. Therefore, the problem (10) is equivalent to maximizing every term in the summation subject to the corresponding constraint, i.e., it can be represented as $K$ independent problems shown as

$$
\begin{aligned}
\max _{\dot{\boldsymbol{\delta}}_{k}} & \left(2\left|\beta_{k}\right|^{2} \operatorname{tr}\left[\dot{\tilde{\mathbf{H}}}_{k}^{H} \mathbf{Q}^{-1} \dot{\tilde{\mathbf{H}}}_{k} \mathbf{R}_{\mathbf{S}}\right]\right)^{-1}, k=1,2, \cdots, K \\
\text { s.t. } & \left\|\dot{\boldsymbol{\delta}}_{k}\right\|_{F} \leq \sigma_{k}
\end{aligned}
$$


Note that the optimization variables in the problem above are complex. In order to recast the problem (11) as a convex one, we can reformulate this problem as a problem with real variables in Appendix B, which can be illustrated as

$$
\begin{aligned}
\max _{\dot{\delta}_{R, k}} & \left(\left|\beta_{k}\right|^{2} \operatorname{tr}\left[\dot{\tilde{\mathbf{H}}}_{R, k}^{T} \mathbf{Q}_{R}^{-1} \dot{\tilde{\mathbf{H}}}_{R, k} \mathbf{R}_{R, \mathbf{S}}\right]\right)^{-1} \\
\text { s.t. } & \left\|\dot{\boldsymbol{\delta}}_{R, k}\right\|_{F} \leq \gamma_{k}
\end{aligned}
$$

where $\dot{\tilde{\mathbf{H}}}_{R, k}, \mathbf{R}_{R, \mathbf{S}}$, and $\dot{\boldsymbol{\delta}}_{R, k}$ are defined in (31), $\gamma_{k}$ in (37), and

$$
\mathbf{Q}_{R}=\left[\begin{array}{ll}
\mathbf{Q}_{x} & -\mathbf{Q}_{y} \\
\mathbf{Q}_{y} & \mathbf{Q}_{x}
\end{array}\right]
$$

$\mathbf{Q}_{x}=\operatorname{Re}(\mathbf{Q}), \mathbf{Q}_{y}=\operatorname{Im}(\mathbf{Q})$. Because $\mathbf{Q}$ is positive definite, according to [17], $\mathbf{Q}_{R}$ is also positive definite symmetric matrix.

It is obvious that the term $\operatorname{tr}\left(\dot{\tilde{\mathbf{H}}}_{R, k}^{T} \mathbf{Q}_{R}^{-1} \dot{\tilde{\mathbf{H}}}_{R, k} \mathbf{R}_{R, \mathbf{S}}\right)$ is convex with respect to $\dot{\boldsymbol{\delta}}_{R, k}[16]$, and then (12) is equivalent to minimizing this term with respect to $\dot{\delta}_{R, k}$, which can be equivalently represented as

$$
\begin{array}{ll}
\min _{\dot{\delta}_{R, k}, t} & t \\
\text { s.t. } & \operatorname{tr}\left(\mathbf{T}^{T} \dot{\tilde{\mathbf{H}}}_{R, k}^{T} \mathbf{Q}_{R}^{-1} \dot{\tilde{\mathbf{H}}}_{R, k} \mathbf{T}\right) \leq t \\
& \left\|\dot{\boldsymbol{\delta}}_{R, k}\right\|_{F} \leq \gamma_{k}
\end{array}
$$

where $\mathbf{T}=\mathbf{R}_{R, \mathbf{S}}^{1 / 2}$, i.e., $\mathbf{T}$ is the square root of $\mathbf{R}_{R, \mathbf{S}}$ [16], and $t$ is an auxiliary variable.

Due to the fact that $\operatorname{tr}(\mathbf{A B C D})=(\operatorname{vec}(\mathbf{D}))^{T}\left(\mathbf{A} \otimes \mathbf{C}^{T}\right) \operatorname{vec}\left(\mathbf{B}^{T}\right)$, we can obtain

$$
\begin{aligned}
\operatorname{tr}\left(\mathbf{T}^{T} \dot{\tilde{\mathbf{H}}}_{R, k}^{T} \mathbf{Q}_{R}^{-1} \dot{\tilde{\mathbf{H}}}_{R, k} \mathbf{T}\right) & =\left(\operatorname{vec}\left(\dot{\tilde{\mathbf{H}}}_{R, k} \mathbf{T}\right)\right)^{T}\left(\mathbf{I} \otimes\left(\mathbf{Q}_{R}^{-1}\right)^{T}\right) \operatorname{vec}\left(\left(\mathbf{T}^{T} \dot{\tilde{\mathbf{H}}}_{R, k}^{T}\right)^{T}\right) \\
& =\left(\operatorname{vec}\left(\dot{\tilde{\mathbf{H}}}_{R, k} \mathbf{T}\right)\right)^{T}\left(\mathbf{I} \otimes \mathbf{Q}_{R}\right)^{-1} \operatorname{vec}\left(\dot{\tilde{\mathbf{H}}}_{R, k} \mathbf{T}\right)
\end{aligned}
$$

Using Schur's Complement [16, pp.472] and (15), the problem (14) can be recast as an SDP

$$
\begin{aligned}
\min _{\dot{\delta}_{R, k}, t} t & \\
\text { s.t. } & {\left[\begin{array}{cc}
t & \left(\operatorname{vec}\left(\dot{\tilde{\mathbf{H}}}_{R, k} \mathbf{T}\right)\right)^{T} \\
\operatorname{vec}\left(\dot{\tilde{\mathbf{H}}}_{R, k} \mathbf{T}\right) & \mathbf{I} \otimes \mathbf{Q}_{R}
\end{array}\right] \pm \mathbf{0} } \\
& {\left[\begin{array}{cc}
\gamma_{k}^{2} & \dot{\boldsymbol{\delta}}_{R, k}^{T} \\
\dot{\boldsymbol{\delta}}_{R, k} & \mathbf{I}
\end{array}\right] \pm \mathbf{0} }
\end{aligned}
$$

Substituting $\left\{\dot{\boldsymbol{\delta}}_{R, k}\right\}_{k=1}^{K}$ obtained from (16) into (8), $\mathbf{R}_{\mathbf{S}}$ can be solved by an SDP

$$
\begin{aligned}
\min _{\mathbf{R}_{\mathbf{S}}, t} t & \\
\text { s.t. } & {\left[\begin{array}{cc}
t & (\operatorname{vec}(\mathbf{I}))^{T} \\
\operatorname{vec}(\mathbf{I}) & \mathbf{I} \otimes \mathbf{F}_{11}
\end{array}\right] \pm \mathbf{0} } \\
& \operatorname{tr}\left(\mathbf{R}_{\mathbf{S}}\right)=L P \\
& \mathbf{R}_{\mathbf{S}} \pm \mathbf{0}
\end{aligned}
$$

So far, we know how to solve $\dot{\boldsymbol{\delta}}_{k}$ for fixed $\mathbf{R}_{\mathbf{S}}$, and $\mathbf{R}_{\mathbf{s}}$ for fixed $\dot{\boldsymbol{\delta}}_{k}$. Similar to Algorithm 3 proposed in [9], an iterative algorithm is proposed to improve the worst-case performance of parameter estimation, which is shown as follows. 
Algorithm: Given an initial value of the WCM, $\dot{\boldsymbol{\delta}}_{k}$ and $\mathbf{R}_{\mathbf{S}}$ in the case of the Traceopt criterion can be optimized by repeating the following steps:

1. Solve (16) to obtain the optimum $\dot{\boldsymbol{\delta}}_{k}$.

2. Substituting $\dot{\boldsymbol{\delta}}_{k}$ into (8), solve (17) to obtain $\mathbf{R}_{\mathbf{s}}$.

3. Go to step 1 until the CRB decrease becomes insignificant.

Using many well-known algorithms (see, e.g., [13]), the problems (16), (17) can be solved very efficiently. It is noted that the proposed method can only obtain the WCM other than the ultimate transmitted waveforms. In practice, the ultimate waveforms can be asymptotically synthesized by using the method in [18].

\section{Numerical Examples}

In this section, several numerical simulations are given to demonstrate the benefits of the proposed method, as compared to uncorrelated waveforms. Similar to that in [7], uncorrelated waveforms can be generated by using Hadamard codes with $K=256$ samples for each transmitted pulse which are orthogonal to each other.

Consider the following two MIMO radar systems with $M_{t}=3$ transmitting elements and $M_{r}=3$ receiving elements possessing various antenna configurations: MIMO radar $(0.5,0.5)$, and MIMO radar $(1.5,0.5)$, where the parameters specifying each radar system are the inter-element spacing of the transmitter and receiver (in units of wavelengths), respectively. The number of snapshots is $L=256$. The array signal-to-noise ratio (ASNR) defined as $P M_{t} M_{r} / \sigma_{\mathrm{w}}^{2}$ varies from -10 to $30 \mathrm{~dB}$, where $P$ stands for the total transmitted power, and $\sigma_{\mathrm{W}}^{2}$ denotes the variance of the additive white thermal noise. There is a strong jammer at $-5^{\circ}$ with an array-interference-to-noise ratio (AINR) defined as the product of the incident interference power and $M_{r}$ divided by $\sigma_{\mathrm{w}}^{2}$, equal to $60 \mathrm{~dB}$. In the following numerical examples, there is only one target with unit amplitude at $\theta=20^{\circ}$ in the considered range bin.

It is known from Section II that the CRB must be estimated using the initial location parameter estimate. There are many methods for estimating this parameter (see, e.g., [19] and the reference therein for more details).

In the following examples, we examine the effectiveness of the proposed method in the case of only existing the initial angle estimate error. In this scenario, we assume that the initial angle estimate has an uncertainty $\Delta \theta=\left[-3^{\circ}, 3^{\circ}\right]$, i.e., $\tilde{\theta}=\left[17^{\circ}, 23^{\circ}\right]$, where $\tilde{\theta}$ is the estimate of $\theta$. After calculating, we can obtain $\sigma=9.8962$ for $\operatorname{MIMO}(0.5,0.5)$, and $\sigma=32.2755$ for MIMO $(1.5,0.5)$.

Figure 1 shows the optimal transmit beampatterns obtained by the proposed method with $\mathrm{ASNR}=10 \mathrm{~dB}$. One can see that the peak of the transmit beampattern is placed around the target location, which means that the worst-case performance in the convex uncertainty can be improved. Moreover, there are grating lobes of the peak in the case of MIMO radar $(1.5,0.5)$ shown in Figure 1 (b) due to the sparse transmit array. 

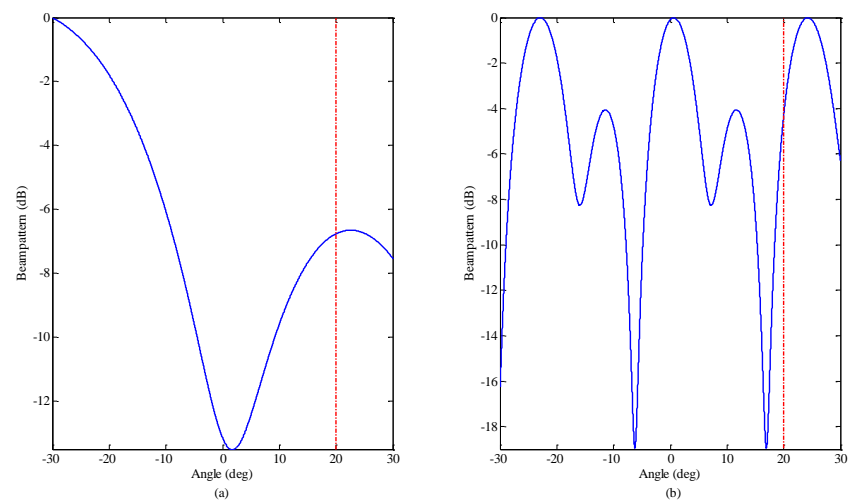

Figure 1. Optimal Transmit Beampatterns Obtained by the Proposed Method with ASNR=10 Db. (a) Optimal Transmit Beampatterns for MIMO Radar (0.5, 0.5). (b) Optimal Transmit Beampatterns for MIMO Radar $(1.5,0.5)$

In Figure 2, the worst-case CRB obtained by the proposed method versus ASNR is showed, as compared to that of uncorrelated waveforms. It is obvious that the CRB decreases as the increase of ASNR. Moreover, one can observe that the transmitted waveforms obtained by the proposed method have a better worst-case performance of parameter estimation than uncorrelated waveforms. Furthermore, the CRB obtained by the proposed method is asymptotically identical to that of uncorrelated waveforms as ASNR increases, which is similar to that in [11] (see this reference for explanation.). Also, it is noted that the CRB obtained for MIMO radar $(1.5,0.5)$ shown in Figure 2 (b) is lower than that for MIMO radar $(0.5,0.5)$ shown in Figure 2 (a), which is due to the virtual receiving aperture for the former is larger than that for the latter [2].
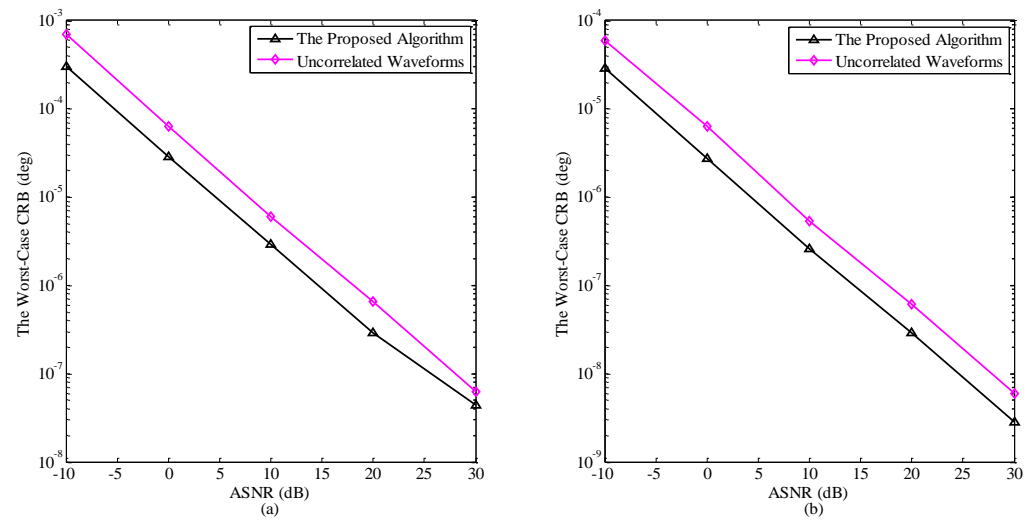

Figure 2. The Worst-Case CRB Obtained by the Proposed Method versus a SNR, as Well as that of Uncorrelated Waveforms. (a) CRB for MIMO Radar $(0.5,0.5)$. (b) CRB for MIMO Radar $(1.5,0.5)$

\section{Conclusions}

In this paper, we have investigated the problem of robust MIMO radar waveform optimization to improve the worst-case performance of parameter estimation by explicitly incorporating the uncertainty in the parameters error into the optimization model, which is based on the constrained CRB. An iterative algorithm has been proposed for jointly optimizing the transmitted waveforms and the perturbation of the derivation of the target channel matrix, each step in which can be relaxed as an SDP by using some matrix 
inequality, and hence it can be solved efficiently. Numerical examples have shown that the proposed algorithm lowers the worst-case CRB very obviously compared to uncorrelated waveforms.

\section{Acknowledgements}

This work is sponsored in part by NSFC under Grant 61401526, 61301258, 61103143 and the Education Department of Henan Province Natural Science Research Program under Grant 14B510033.

\section{Appendix A}

\section{Constrained Cramero-Rao Bound}

Based on the signal model in (1), in the following, we derive the constrained CRB of the unknown target parameters $\boldsymbol{\theta}=\left[\theta_{1}, \theta_{2}, \cdots, \theta_{K}\right]^{T}$. According to [14], the constrained CRB can be written as

$$
\mathbf{C}_{C C R B}=\mathbf{U}\left(\mathbf{U}^{H} \mathbf{F} \mathbf{U}\right)^{-1} \mathbf{U}^{H}
$$

where $\mathbf{U}$ satisfies

$$
\mathbf{G}(\mathbf{x}) \mathbf{U}(\mathbf{x})=\mathbf{0}, \quad \mathbf{U}^{H}(\mathbf{x}) \mathbf{U}(\mathbf{x})=\mathbf{I}
$$

in which $G(\mathbf{x})=\frac{\partial \mathbf{g}(\mathbf{x})}{\partial \mathbf{x}}$ is assumed to have full row rank, and $\mathbf{U}$ is the tangent hyperplane of $\mathbf{g}(\mathbf{x})$ that is equality constraint set on $\mathbf{x}$. In addition, $\mathbf{F}$ is the Fisher Information Matrix (FIM) with respect to $\mathbf{x}=\left[\boldsymbol{\theta}^{T}, \boldsymbol{\beta}_{R}^{T}, \boldsymbol{\beta}_{I}^{T}\right]^{T}$, where $\boldsymbol{\beta}_{R}=\left[\beta_{R, 1}, \beta_{R, 2}, \cdots, \beta_{R, K}\right]^{T}, \boldsymbol{\beta}_{I}=\left[\beta_{I, 1}, \beta_{I, 2}, \cdots, \beta_{I, K}\right]^{T}, \beta_{R}=\operatorname{Re}(\beta)$, and $\beta_{I}=\operatorname{Im}(\beta)$.

Following [14], some prior information can be available in array signal processing, for example, constant modulus constraint on the transmitted waveform, etc.. Here, the complex amplitude matrix $\boldsymbol{\beta}=\operatorname{diag}\left(\beta_{1}, \beta_{2}, \cdots, \beta_{K}\right)$ can be assumed to be known as

$$
\begin{aligned}
& g_{i}(\mathbf{x})=\beta_{R, i}-1=0, \quad i=1, \cdots, K \\
& g_{j}(\mathbf{x})=\beta_{I, j}-1=0, \quad j=K+1, \cdots, 2 K
\end{aligned}
$$

Therefore, $\mathbf{G}(\mathbf{x})=\frac{\partial \mathbf{g}(\mathbf{x})}{\partial \mathbf{x}}$ has the form $\mathbf{G}=\left[\mathbf{0}_{2 K \times K}, \mathbf{I}_{2 K \times 2 K}\right]$ with $\mathbf{0}_{2 K \times K}$ denoting a zero matrix of size $2 K \times K$, and the corresponding null space $\mathbf{U}$ can be represented as

$$
\mathbf{U}=\left[\begin{array}{ll}
\mathbf{I}_{K \times K} & \mathbf{0}_{K \times 2 K}
\end{array}\right]^{H}
$$

In what follows, we will calculate the FIM with respect to $\mathbf{x}$ (Here we only consider one-dimensional targets.). According to [15], we can obtain

$$
\mathbf{F}\left(x_{i}, x_{j}\right)=2 \operatorname{Re}\left\{\operatorname{tr}\left[\frac{\partial\left(\sum_{k=1}^{K} \beta_{k} \mathbf{H}_{k} \mathbf{S}\right)^{H}}{\partial x_{i}} \mathbf{Q}^{-1} \frac{\partial\left(\sum_{k=1}^{K} \beta_{k} \mathbf{H}_{k} \mathbf{S}\right)}{\partial x_{j}}\right]\right\}
$$

And then

$$
\begin{aligned}
\mathbf{F}\left(\theta_{i}, \theta_{j}\right) & =2 \operatorname{Re}\left\{\operatorname{tr}\left[\frac{\partial\left(\sum_{k=1}^{K} \beta_{k} \mathbf{H}_{k} \mathbf{S}\right)^{H}}{\partial \boldsymbol{\theta}_{i}} \mathbf{Q}^{-1} \frac{\partial\left(\sum_{k=1}^{K} \beta_{k} \mathbf{H}_{k} \mathbf{S}\right)}{\partial \boldsymbol{\theta}_{j}}\right]\right\} \\
& =2 \operatorname{Re} \operatorname{tr}\left[\beta_{i}^{*} \beta_{j} \dot{\mathbf{H}}_{i}^{H} \mathbf{Q}^{-1} \dot{\mathbf{H}}_{j} \mathbf{R}_{\mathbf{S}}\right]
\end{aligned}
$$


Hence, $\mathbf{F}(\boldsymbol{\theta}, \boldsymbol{\theta})=2 \operatorname{Re}\left(\mathbf{F}_{11}\right)$ with $\mathbf{F}_{11}$ given in (5).

Similar to [7], we have

$$
\mathbf{F}^{T}\left(\boldsymbol{\beta}_{R}, \boldsymbol{\theta}\right)=\mathbf{F}\left(\boldsymbol{\theta}, \boldsymbol{\beta}_{R}\right)=2 \operatorname{Re}\left(\mathbf{F}_{12}\right)
$$

and

$$
\mathbf{F}^{T}\left(\boldsymbol{\beta}_{I}, \boldsymbol{\theta}\right)=\mathbf{F}\left(\boldsymbol{\theta}, \boldsymbol{\beta}_{I}\right)=-2 \operatorname{Im}\left(\mathbf{F}_{12}\right)
$$

where $\left[\mathbf{F}_{12}\right]_{i j}=\operatorname{tr}\left[\beta_{i}^{*} \dot{\mathbf{H}}_{i}^{H} \mathbf{Q}^{-1} \mathbf{H}_{j} \mathbf{R}_{\mathbf{S}}\right]$.

Also,

$$
\mathbf{F}\left(\boldsymbol{\beta}_{R}, \boldsymbol{\beta}_{R}\right)=\mathbf{F}\left(\boldsymbol{\beta}_{I}, \boldsymbol{\beta}_{I}\right)=2 \operatorname{Re}\left(\mathbf{F}_{22}\right)
$$

and

$$
\mathbf{F}\left(\boldsymbol{\beta}_{R}, \boldsymbol{\beta}_{I}\right)=\mathbf{F}^{T}\left(\boldsymbol{\beta}_{I}, \boldsymbol{\beta}_{R}\right)=-2 \operatorname{Im}\left(\mathbf{F}_{22}\right)
$$

where $\left[\mathbf{F}_{22}\right]_{i j}=\operatorname{tr}\left[\mathbf{H}_{i}^{H} \mathbf{Q}^{-1} \mathbf{H}_{j} \mathbf{R}_{\mathbf{s}}\right]$.

Based on the discussion above, the FIM $\mathbf{F}$ with respect to $\mathbf{x}$ can be expressed as

$$
\mathbf{F}=2\left[\begin{array}{ccc}
\operatorname{Re}\left(\mathbf{F}_{11}\right) & \operatorname{Re}\left(\mathbf{F}_{12}\right) & -\operatorname{Im}\left(\mathbf{F}_{12}\right) \\
\operatorname{Re}^{T}\left(\mathbf{F}_{12}\right) & \operatorname{Re}\left(\mathbf{F}_{22}\right) & -\operatorname{Im}\left(\mathbf{F}_{22}\right) \\
-\operatorname{Im}^{T}\left(\mathbf{F}_{12}\right) & -\operatorname{Im}^{T}\left(\mathbf{F}_{22}\right) & \operatorname{Re}\left(\mathbf{F}_{22}\right)
\end{array}\right]
$$

With (18), (21), (5), and (24)-(28), (3) can be obtained immediately.

\section{Appendix B}

\section{Derivation of (12)}

To simplify notation, we define

$$
\begin{aligned}
& \dot{\mathbf{H}}_{k, x}=\operatorname{Re}\left(\dot{\mathbf{H}}_{k}\right), \dot{\mathbf{H}}_{k, y}=\operatorname{Im}\left(\dot{\mathbf{H}}_{k}\right), \dot{\tilde{\mathbf{H}}}_{k, x}=\operatorname{Re}\left(\dot{\tilde{\mathbf{H}}}_{k}\right), \dot{\tilde{\mathbf{H}}}_{k, y}=\operatorname{Im}\left(\dot{\tilde{\mathbf{H}}}_{k}\right), \dot{\boldsymbol{\delta}}_{k, x}=\operatorname{Re}\left(\dot{\boldsymbol{\delta}}_{k}\right) \\
& \dot{\boldsymbol{\delta}}_{k, y}=\operatorname{Im}\left(\dot{\boldsymbol{\delta}}_{k}\right), \mathbf{R}_{\mathbf{s}, x}=\operatorname{Re}\left(\mathbf{R}_{\mathbf{s}}\right), \mathbf{R}_{\mathbf{s}, y}=\operatorname{Im}\left(\mathbf{R}_{\mathbf{s}}\right), \mathbf{Z}_{x}=\operatorname{Re}(\mathbf{Z}), \mathbf{Z}_{y}=\operatorname{Im}(\mathbf{Z})
\end{aligned}
$$

where $\mathbf{Z}=\mathbf{Q}^{-1}$.

Then

$$
\begin{aligned}
& \dot{\tilde{\mathbf{H}}}_{k}^{H} \mathbf{Q}^{-1} \dot{\tilde{\mathbf{H}}}_{k} \mathbf{R}_{\mathbf{S}}=\left(\dot{\tilde{\mathbf{H}}}_{k, x}+j \dot{\tilde{\mathbf{H}}}_{k, y}\right)^{H}\left(\mathbf{Z}_{x}+j \mathbf{Z}_{y}\right)\left(\dot{\tilde{\mathbf{H}}}_{k, x}+j \dot{\tilde{\mathbf{H}}}_{k, y}\right)\left(\mathbf{R}_{\mathbf{s}, x}+j \mathbf{R}_{\mathbf{S}, y}\right) \\
& =\left(\dot{\tilde{\mathbf{H}}}_{k, x}^{T} \mathbf{Z}_{x} \dot{\tilde{\mathbf{H}}}_{k, x}+j \dot{\tilde{\mathbf{H}}}_{k, x}^{T} \mathbf{Z}_{y} \dot{\tilde{\mathbf{H}}}_{k, x}-j \dot{\tilde{\mathbf{H}}}_{k, y}^{T} \mathbf{Z}_{x} \dot{\tilde{\mathbf{H}}}_{k, x}+\dot{\tilde{\mathbf{H}}}_{k, y}^{T} \mathbf{Z}_{y} \dot{\tilde{\mathbf{H}}}_{k, x}+j \dot{\tilde{\mathbf{H}}}_{k, x}^{T} \mathbf{Z}_{x} \dot{\tilde{\mathbf{H}}}_{k, y}\right. \\
& \left.-\dot{\tilde{\mathbf{H}}}_{k, x}^{T} \mathbf{Z} \dot{\tilde{\mathbf{H}}}_{k, y}+\dot{\tilde{\mathbf{H}}}_{k, y}^{T} \mathbf{Z} \dot{\tilde{\mathbf{H}}}_{k, y}+j \dot{\tilde{\mathbf{H}}}_{k, y}^{T} \mathbf{Z}_{y,} \dot{\tilde{\mathbf{H}}}_{k, y}\right)\left(\mathbf{R}_{\mathrm{s}, x}+j \mathbf{R}_{\mathrm{s}, y}\right) \\
& =\dot{\tilde{\mathbf{H}}}_{k, x}^{T} \mathbf{Z}_{x} \dot{\tilde{\mathbf{H}}}_{k, x} \mathbf{R}_{\mathbf{S}, x}-\dot{\tilde{\mathbf{H}}}_{k, x}^{T} \mathbf{Z} \dot{\tilde{\mathbf{H}}}_{k, y} \mathbf{R}_{\mathbf{s}, x}+\dot{\tilde{\mathbf{H}}}_{k, y}^{T} \mathbf{Z}_{x} \dot{\tilde{\mathbf{H}}}_{k, y} \mathbf{R}_{\mathbf{s}, x}+\dot{\tilde{\mathbf{H}}}_{k, y}^{T} \mathbf{Z}_{y} \dot{\tilde{\mathbf{H}}}_{k, x} \mathbf{R}_{\mathbf{s}, x} \\
& -\dot{\tilde{\mathbf{H}}}_{k, x}^{T} \mathbf{Z}_{y} \dot{\tilde{\mathbf{H}}}_{k, x} \mathbf{R}_{\mathbf{S}, y}+\dot{\tilde{\mathbf{H}}}_{k, y}^{T} \mathbf{Z}_{x} \dot{\tilde{\mathbf{H}}}_{k, x} \mathbf{R}_{\mathbf{s}, y}-\dot{\tilde{\mathbf{H}}}_{k, x}^{T} \mathbf{Z}_{x} \dot{\tilde{\mathbf{H}}}_{k, y} \mathbf{R}_{\mathbf{s}, y}-\dot{\tilde{\mathbf{H}}}_{k, y}^{T} \mathbf{Z}_{y,} \dot{\mathbf{H}}_{k, y} \mathbf{R}_{\mathbf{s}, y}
\end{aligned}
$$

Let

Then we have

$$
\begin{aligned}
\dot{\mathbf{H}}_{R, k} & =\left[\begin{array}{ll}
\dot{\mathbf{H}}_{k, x} & -\dot{\mathbf{H}}_{k, y} \\
\dot{\mathbf{H}}_{k, y} & \dot{\mathbf{H}}_{k, x}
\end{array}\right], \dot{\tilde{\mathbf{H}}}_{R, k}=\left[\begin{array}{cc}
\dot{\tilde{\mathbf{H}}}_{k, x} & -\dot{\tilde{\mathbf{H}}}_{k, y} \\
\dot{\tilde{\mathbf{H}}}_{k, y} & \dot{\tilde{\mathbf{H}}}_{k, x}
\end{array}\right], \dot{\boldsymbol{\delta}}_{R, k}=\left[\begin{array}{cc}
\dot{\boldsymbol{\delta}}_{k, x} & -\dot{\boldsymbol{\delta}}_{k, y} \\
\dot{\boldsymbol{\delta}}_{k, y} & \dot{\boldsymbol{\delta}}_{k, x}
\end{array}\right] \\
\mathbf{R}_{R, \mathbf{s}} & =\left[\begin{array}{ll}
\mathbf{R}_{\mathbf{S}, x} & -\mathbf{R}_{\mathbf{S}, y} \\
\mathbf{R}_{\mathbf{S}, y} & \mathbf{R}_{\mathbf{S}, x}
\end{array}\right], \mathbf{Z}_{R}=\left[\begin{array}{cc}
\mathbf{Z}_{x} & -\mathbf{Z}_{y} \\
\mathbf{Z}_{y} & \mathbf{Z}_{x}
\end{array}\right]
\end{aligned}
$$




$$
\begin{aligned}
& \dot{\tilde{\mathbf{H}}}_{R, k}^{T} \mathbf{Z}_{R} \dot{\tilde{\mathbf{H}}}_{R, k} \mathbf{R}_{R, \mathbf{S}}=\left[\begin{array}{cc}
\dot{\tilde{\mathbf{H}}}_{k, x} & -\dot{\tilde{\mathbf{H}}}_{k, y} \\
\dot{\tilde{\mathbf{H}}}_{k, y} & \dot{\tilde{\mathbf{H}}}_{k, x}
\end{array}\right]^{T}\left[\begin{array}{cc}
\mathbf{Z}_{x} & -\mathbf{Z}_{y} \\
\mathbf{Z}_{y} & \mathbf{Z}_{x}
\end{array}\right]\left[\begin{array}{cc}
\dot{\tilde{\mathbf{H}}}_{k, x} & -\dot{\tilde{\mathbf{H}}}_{k, y} \\
\dot{\tilde{\mathbf{H}}}_{k, y} & \dot{\tilde{\mathbf{H}}}_{k, x}
\end{array}\right]\left[\begin{array}{cc}
\mathbf{R}_{\mathbf{S}, x} & -\mathbf{R}_{\mathbf{S}, y} \\
\mathbf{R}_{\mathbf{S}, y} & \mathbf{R}_{\mathbf{S}, x}
\end{array}\right] \\
& =2\left(\dot{\tilde{\mathbf{H}}}_{k, x}^{T} \mathbf{Z}_{x} \dot{\tilde{\mathbf{H}}}_{k, x} \mathbf{R}_{\mathbf{S}, x}-\dot{\tilde{\mathbf{H}}}_{k, x}^{T} \mathbf{Z}_{y} \dot{\tilde{\mathbf{H}}}_{k, y} \mathbf{R}_{\mathbf{s}, x}+\dot{\tilde{\mathbf{H}}}_{k, y}^{T} \mathbf{Z}_{x} \dot{\tilde{\mathbf{H}}}_{k, y} \mathbf{R}_{\mathbf{s}, x}+\dot{\tilde{\mathbf{H}}}_{k, y}^{T} \mathbf{Z}_{y} \dot{\tilde{\mathbf{H}}}_{k, x} \mathbf{R}_{\mathbf{S}, x}\right. \\
& \left.-\dot{\tilde{\mathbf{H}}}_{k, x}^{T} \mathbf{Z}_{y} \dot{\tilde{\mathbf{H}}}_{k, x} \mathbf{R}_{\mathbf{s}, y}+\dot{\tilde{\mathbf{H}}}_{k, y}^{T} \mathbf{Z}_{x} \dot{\tilde{\mathbf{H}}}_{k, x} \mathbf{R}_{\mathbf{s}, y}-\dot{\tilde{\mathbf{H}}}_{k, x}^{T} \mathbf{Z}_{x} \dot{\tilde{\mathbf{H}}}_{k, y} \mathbf{R}_{\mathbf{s}, y}-\dot{\tilde{\mathbf{H}}}_{k, y}^{T} \mathbf{Z}_{y} \dot{\tilde{\mathbf{H}}}_{k, y} \mathbf{R}_{\mathbf{s}, y}\right)
\end{aligned}
$$

Therefore, (12) holds immediately.

Besides, because

$$
\begin{array}{r}
\dot{\tilde{\mathbf{H}}}_{R, k}=\dot{\mathbf{H}}_{R, k}+\dot{\boldsymbol{\delta}}_{R, k} \\
\dot{\boldsymbol{\delta}}_{R, k}=\left[\begin{array}{cc}
\dot{\boldsymbol{\delta}}_{k, x} & -\dot{\boldsymbol{\delta}}_{k, y} \\
\dot{\boldsymbol{\delta}}_{k, y} & \dot{\boldsymbol{\delta}}_{k, x}
\end{array}\right]
\end{array}
$$

and

we can obtain

$$
\left\|\dot{\boldsymbol{\delta}}_{k}\right\|_{F} \leq \sigma_{k}
$$

$$
\left\|\dot{\boldsymbol{\delta}}_{R, k}\right\|_{F} \leq \gamma_{k}
$$

where

$$
\gamma_{k}=\sqrt{2} \sigma_{k}
$$

\section{References}

[1] J. Li and P. Stoica, "MIMO radar with colocated antennas", IEEE Signal Processing Magazine, vol. 24, no. 5, (2007), pp. 106-114.

[2] E. Fishler, A. Haimovich, R. Blum, L. Cimini, D. Chizhik and R. Valenzuela, "Spatial diversity in radars-models and detection performance", IEEE Trans. on Signal Processing, vol. 54, no. 3, (2006), pp. 823-838.

[3] J. Li, P. Stoica, L. Xu and W. Roberts, "On parameter identifiability of MIMO radar", IEEE Trans. on Signal Processing Lett., vol. 14, no. 12, (2007), pp. 968-971.

[4] D. R. Fuhrmann and G. S. Antonio, "Transmit beamforming for MIMO radar systems using signal cross-correlation", IEEE Trans. on Aerospace and Electronic System, vol. 44, no. 1, (2008), pp. 171186.

[5] G. Cui, H. Li and M. Rangaswamy, "MIMO Radar Waveform Design With Constant Modulus and Similarity Constraints", IEEE Trans. on Signal Processing, vol. 62, no. 2, (2014), pp. 343-353.

[6] C. Y. Chen and P. P. Vaidyanathan, "MIMO Radar Ambiguity Properties and Optimization Using Frequency-Hopping Waveforms", IEEE Trans. on Signal Processing, vol. 56, no. 12, (2008), pp. 59265936.

[7] J. Li, L. Xu, P. Stoica, K. W. Forsythe and D. W. Bliss, "Range Compression and Waveform Optimization for MIMO Radar: A Cramer-Rao Bound Based Study”, IEEE Trans. on Signal Processing, vol. 56, no. 1, (2008), pp. 218-232.

[8] B. Friedlander, "Waveform Design for MIMO Radars", IEEE Trans. on Aerospace and Electronic Systems, vol. 43, no. 3, (2007), pp. 1227-1238.

[9] C. Y. Chen, P. P. Vaidyanathan. MIMO Radar Waveform Optimization with Prior Information of the Extended Target and Clutter. IEEE Trans. on Signal Processing, 57 (9): 3533-3544 (2009).

[10] Bo Tang, Jun Tang, Yingning Pen. Waveform Optimization for MIMO Radar in Colored Noise: Further Results for Estimation-Oriented Criteria. IEEE Trans. on Signal Processing, 60 (3):1517-1522 (2012).

[11] T. Naghibi, M. Namvar, F. Behnia. Optimal and robust waveform design for MIMO radars in the presence of clutter. Signal Processing, 90 (4): 1103-1117 (2010).

[12] Jun Liu, Hongbin Li, and Braham Himed. Joint Optimization of Transmit and Receive Beamforming in Active Arrays. IEEE Trans. on Signal Processing Lett., 21 (1): 39-42 (2014).

[13] A. Ben-Tal, A. Nemirovski, Lectures on Modern Convex Optimization, ser. Optimization. Philadelphia, PA: MPS-SIAM, (2001).

[14] P. Stoica, C. Ng Boon, On the Cramer-Rao Bound Under Parametric Constraints, IEEE Signal Process. Lett., 5: 177 - 179 (1998).

[15] P. Stoica and R. L. Moses, "Spectral Analysis of Signals", NJ: Prentice-Hall, Upper SaddleRiver, (2005).

[16] R. A. Horn and C. R. Johnson, "Matrix Analysis", Cambridge Univ. Press, Cambridge, U.K., (1985).

[17] S. J. Kim, A. Magnani, A. Mutapcic, S. P. Boyd and Z. Q. Luo, "Robust Beamforming via Worst-Case 
SINR Maximization”, IEEE Trans. On Signal Processing, vol. 56, no. 1, (2008), pp. 1539-1547.

[18] P. Stoica, J. Li and X. Zhu, "Waveform Synthesis for Diversity-Based Transmit Beampattern Design", IEEE Trans. On Signal Processing, vol. 56, no. 1, (2008), pp. 2593-2598.

[19] L. Xu, J. Li and P. Stoica, "Adaptive techniques for MIMO radar", The 4th IEEE Workshop on Sensor Array and Multi-Channel Processing, Waltham, MA, (2006), pp. 1-4.

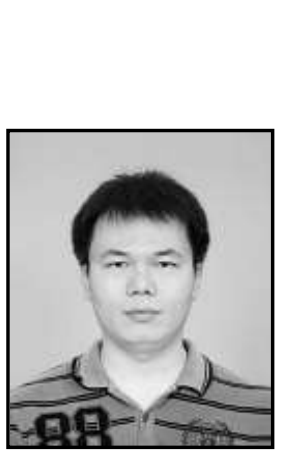

\begin{abstract}
Authors
Yuchun Liu, received the Ph.D. degree from Xidian University in 2013. Dr. Liu joined School of Physics and Electrical Engineering, Zhoukou Normal University in 2013 , where he is currently a lecture. His research interests are mainly in signal processing in passive and MIMO radar, and MIMO radar waveform optimization.
\end{abstract}

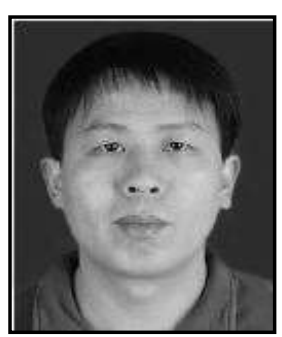

Hai Zhu, received the Ph.D. degree from Xidian University in 2011. Dr. Zhu joined School of Computer Science and Technology, Zhoukou Normal University in 2011, where he is currently an associate professor and assistant dean of the college. His research interests are mainly in array signal processing and MIMO radar waveform optimization.

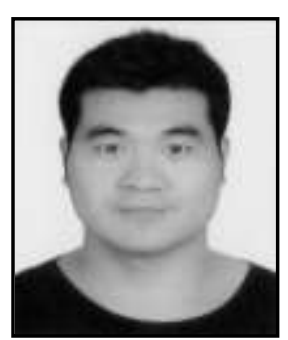

Hongyan Wang, received the Ph.D. degree from Xidian University in 2012. Dr. Wang joined the College of Information Engineering, Dalian University in 2013, where he is currently a lecture. His research interests are mainly in MIMO radar waveform optimization, MIMO communication, space-time adaptive processing, and array signal processing. 
International Journal of Signal Processing, Image Processing and Pattern Recognition Vol. 10, No. 6 (2017) 